\title{
THE WAY FOR DETECTING DEFECTS OF GROUND WIRES OF POWER LiNes BY THE METHOD OF MAgNeTIC SCANNING
}

\author{
Oksana V. Limanovskaya, Vsevolod S. Startsev, \\ Mikhail N. Smirnov, Alexandr V. Lemekh \& Elena N. Kolos
}
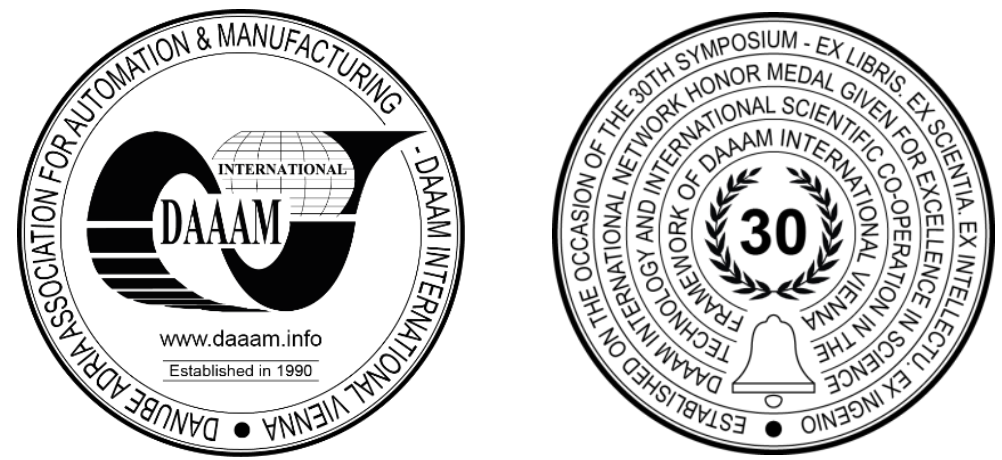

This Publication has to be referred as: Limanovskaya, O[ksana]; Vsevolod, S[tartsev]; Smirnov, M[ikhail]; Lemekh, A[lexandr] \& Kolos, E[lena] (2019). The Way for Detecting Defects of Ground Wires of Power Lines by the Method of Magnetic Scanning, Proceedings of the 30th DAAAM International Symposium, pp.0921-0928, B. Katalinic (Ed.), Published by DAAAM International, ISBN 978-3-902734-22-8, ISSN 1726-9679, Vienna, Austria DOI: $10.2507 / 30$ th.daaam.proceedings. 128

\begin{abstract}
The article presents the system of detection of ground wire defects based on the analysis of loss of metallic area (LMA) and local flaws (LF) diagrams for Cablewalker complex. The system consists of two modules: a module for reading data from a magnetic scanner and a module for analyzing data and generating a report on detected defects. The first module reads the data of LMA and LF diagrams from the magnetic scanner in the form of a binary file and transmits them to the second module. The data analysis module removes noise from the diagrams and identifies the peaks of the diagrams corresponding to the defects of the ground wire. Highlighted peaks are marked in their intensity. To do this, we have entered the colour scheme: green, yellow, red. The green level corresponds to the low intensity of peak, the yellow level is used for average intensity and red level indicates the most intense peaks. Peaks of the red level indicate the most dangerous defects. The system generates a graphical text report containing diagrams with marked peaks and a description of the defects with their location on the ground wire. The system is tested on laboratory data. Test results have shown that the system found all the defects applied to the ground wire. Thus, we have obtained the system for recognition of ground wire defects using the LMA and LF diagrams data.
\end{abstract}

Keywords: analysis of LMA and LD diagram; magnetic scanning; defects of ground wire; signal analysis

\section{Introduction}

At the present time, there are many attempts to create automated systems for the detection of faults in power lines. The development of automated systems is carried out according to two fundamentally different approaches. The first approach uses the general characteristics of the lines to track faults in them [1]. As a rule, these systems analyze the condition of wires [2] or insulators [1] in the system, but cannot detect defects in supports and other elements. Besides, this approach cannot get the exact place of fault in the power line. Therefore, according to the results of the work of these systems, the specialists are required to go online and manually check for the presence of a defect. Thus, this approach, although reducing manual labour, cannot create a fully automated system. The second approach uses different robot 
systems as a cable inspection robot (CIR) with LiDAR [3] or unmanned aerial vehicle (UAV) [4]. This approach has several disadvantages. First, video and image analysis require a lot of computational and time costs. Secondly, the reliability of the detection of a defect strongly depends on the shooting conditions. Thirdly, hidden faults, such as a broken wire core, cannot be visually detected. However, non-destructive testing methods lack these disadvantages [5] and can be successfully used to analyze faults of wires and cables [6].

Magnetic flaw detection is a method of non-destructive testing of cores of steel-aluminium wires and steel ground wires. The ferromagnetic rope or core of a bimetallic wire is magnetized along the longitudinal axis with the help of two magnets made of rare-earth elements, which are mutually opposite, then, using Hall sensors, the values of stray fields caused by defects are read over the surface. The result of the magnetic scanner is a very noisy signal that requires special processing to identify cable defects.

At the present time, The Future Laboratory, together with the Ural Federal University, develop a complex robotic system (Cablewalker) [7] for diagnosis and repair of power lines. Cablewalker is a new technology of digital diagnostics, maintenance and local repair of elements without switching off the power line. The complex consists of two parts: the robotic flying platform and simulator. In the simulator operator plans the trajectory of the movement of the device, objects and control technology - creates a diagnostic mission. The mission passed in the Simulator is saved by the operator and loaded into the on-board computer of the System. According to the mission, CableWalker performs the flight task and stores the diagnostic results in the database (video data, laser, magnetic scanning).

The robotic flying platform is remotely operated vehicles (ROVs) with video, photo and laser cameras installed on it, as well as a magnetic scanner. A wire rope flaw detector INTROS uses as a magnetic scanner. It is developed in the Russian Federation with its own software Wintros. However, the Wintros does not have an open API and cannot be built into a higher-level system, for example, into an expert system, which makes it unsuitable for building an intelligent complex of fault analysis of power lines.

The goal of this work is the development of software with an open interface for reading data from the magnetic scanner Intron and signal processing to identify ground wire defects. This software will be used for the complex diagnostic system of Cablewalker.

\section{The raw data obtaining}

The raw data have obtained during laboratory tests of Intron on the ground wire with damages.

\subsection{The types of ground ware damages}

There are four basic groups of ground wire damages:

1. deformations (shape changes) in the form of waviness, basket shape, extrusion of wires, strands, core, crushing of strands, creases, bends, etc :;

2. loss of cable (core) cross-section as a result of reduction of steel wire cross-section during their surface and internal wear, corrosion, plastic deformation, chemical exposure, etc .;

3. breaks in the outer and inner wires, core;

4. changes in the structure and strength of steel as a result of thermal effects on the cable (core).

The first three types of damage were applied to the ground wire.

\subsection{Steel wire rope flaw detector INTROS: Work Principles and technical characteristics}

The instrument operation is based on magnetic flux leakage (MFL) principle. The wire portion inside the magnetic head is magnetically saturated with strong rare earth magnets in the longitudinal direction. The magnetic field above the wire surface (flux leakage) remains uniform whilst the wire contains no faults. Coils and/or Hall Effect sensors surround the wire and pick up the signal which is of a constant value. When there are changes in the steel cross-section of the wire or broken wires occur, the magnetic field is distorted and flux leakage increases locally. These irregularities register by creating a signal in the sensors. Signals from the sensors are transmitted to an external basic unit for storage and further processing. 
The instrument consists of a basic unit and magnetic head connected with a special cable 5 or $8 \mathrm{~m}$ in length. Magnetic heads designed to inspect ropes with diameter from 6 to $150 \mathrm{~mm}$. During laboratory tests, the magnetic head moves along the ground wire.

The Intros magnetic flaw detector is connected to a single board computer via a USB Type-A interface, through which the flaw detector receives power and exchanges commands via a virtual COM port with a data rate of 115200 baud. Direct control of the flaw detector, such as starting and stopping the magnetic scanning process, calibrating the device, changing the parameters of the scanned wire or cable, setting the date and time, viewing information on the recorded diagrams and unloading them to an external device, clearing the device's memory, obtaining diagnostic information, is done by transfer to the virtual COM port of commands in ASCII encoding. In response to a received command, the instrument returns a success message or an error code if the command cannot be executed.

\subsection{The view of raw data}

INTROS is the dual-function instrument, i.e. non-destructively measures the loss of metallic area (LMA) and reveals local flaws (LF). LMA is a relative measure of the amount of material missing from a location along the wire rope and is measured by comparing a point with a reference point on the rope that represents the maximum metallic cross-sectional area, as measured with an instrument. LF is a discontinuity in a rope such as a broken wire or a corrosion pit that degrades the integrity of the rope at that position.

The typical LMA and LF diagrams are presented in figure 1.

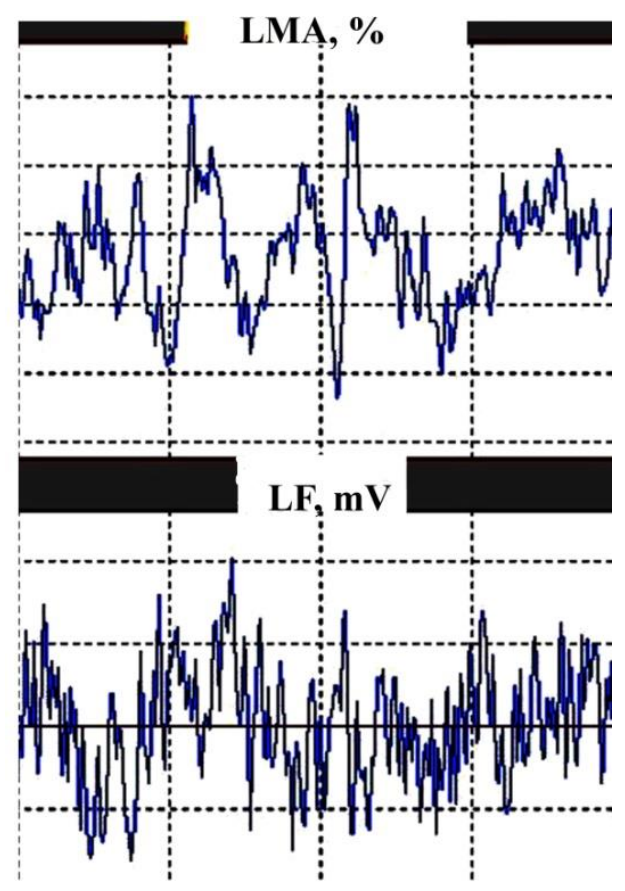

Fig. 1. The examples of LMA and LF diagrams

\subsection{The process of obtaining the magnetic scan data}

During the scanning process, the magnetic scanner is regularly polled by the onboard single-board computer about the distance travelled and free memory space with a second interval. In case the device stops responding, or the distance travelled by the encoder increases without decreasing the amount of unallocated memory, it is assumed that the magnetic scanner has failed. Then the movement on the wire stops and the device restarts. Scanning can be resumed by means the diagnostic panel from the point where it was stopped, and a new file is recorded.

After completion of the passage of the flight of power line, scanning stops. The file is automatically uploaded to its own memory of the onboard single-board computer and to the diagnostic control panel, which provides backup data in case of failure of one of the devices. After the equipment is returned to the office, these files are uploaded to the local data storage. 


\section{Software requirements}

The file uploading system should work on a single-board magnetic scanner computer running the Debian OS. Files should be uploaded to temporary storage in the single-board computer's own memory. It should be possible to upload files to an external database.

The file read from the flaw detector with the record of the wire or ground wire pass must be input to the file analysis system. The output of the system should be the results of data analysis in the form of a list of defects, their location and strength.

Lists of defects with their location and strength are submitted to the input of the report generation system. At the output of this system, a report is generated using the selected template.

In the alpha version of the software, it is possible to combine the file analysis system and the report output system.

\section{Development toolkit alpha version of the program}

In the alpha version of the software, it was decided to combine the file analytic system and the report generation system. For the development of that system, the Python 3.7 language and the framework Anaconda were used. To develop the file uploading system, the $\mathrm{C}++$ language and the framework QT Creator were used.

\section{The file upload automation}

The process of reading magnetic scan data into the memory of a single-plan computer is carried out through a virtual COM port. Through it, a set of commands is sent to control the scanner, including commands for getting a list of files and requesting to transfer a file with a specific index.

File reading is initiated by the command FREAD. When you run the command, the device starts to transfer the contents of the file as binary blocks of 528 bytes, of which 512 bytes of data and 16 bytes of the block header. The transmission is carried out in words of 2 bytes, while the first byte of the word is transmitted first, and for the "direct" packing of the received data into the memory structures of the PC, it is necessary to perform a permutation of even and odd bytes in the packet. Upon completion of a block transfer, the device waits for an ACK control character to transmit the next block, a NAK symbol to retransmit the last block sent, or Esc / Space to interrupt the streaming file. All other commands are ignored.

When receiving an ASCII command to get a list of files, the device returns a list of files in memory with their sequence numbers, as well as the amount of information in the files in bytes. Since there is no data transfer control, it is required to get its size and compare it with the size displayed by the command when uploading the file. Thus, it is possible to determine the case in which the file was received incorrectly (with the loss of part of the content) and repeat the transfer process from scratch.

The developed algorithm for uploading a file with a specified sequence number is as follows. After establishing a connection with the device through the virtual COM port, a command is sent to the device to request a file with the index of the required file. Next, a byte-by-byte response to the buffer is initiated. In the event that, after completion of receiving all bytes waiting on the device side, the buffer contains the first frame of a file of 528 bytes, the next frame is requested, repeated until the final frame is received. Otherwise, the function will return an error code and interrupt the file transfer by sending the space character.

As a result, a binary file containing the parameters of the instrument and a set of points of the recorded channels of LMA and LF diagrams was recorded.

\section{The file analysis system and a report output system}

As can see from figure 1, the LDA and LF diagrams have high noise signal. Therefore, we have to delete this noise signal from raw data. We used 4 filters: Butterworth filter, moving average, weighted local regression, Savitsky-Gaulle filter. The results of using these filters for LMA diagrams are shown in figures 2. 

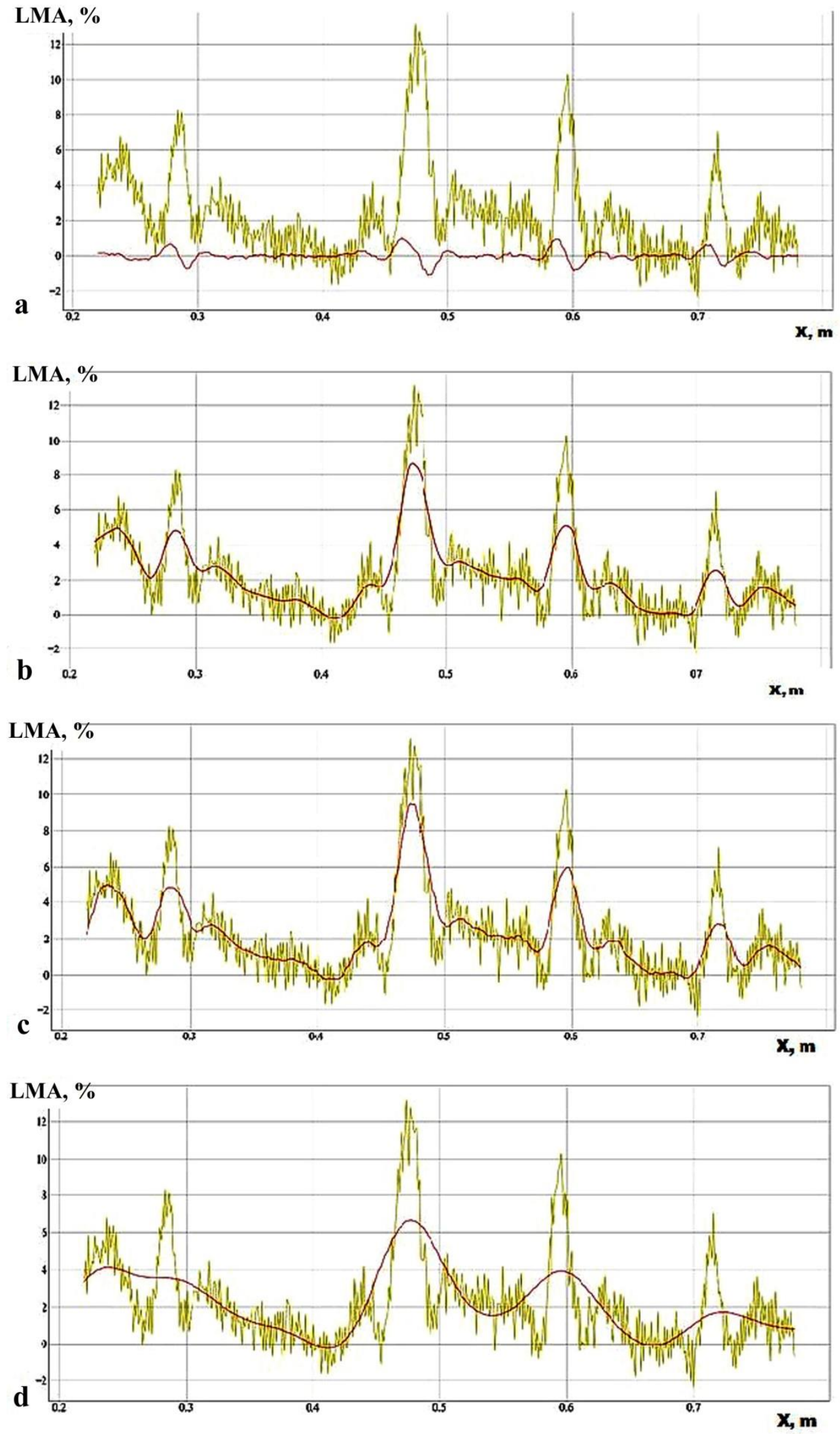

Fig. 2. The results of using filters: (a) Savitsky-Gaulle filter; (b) weighted local regression; (c ) moving average, (d) Butterworth filter 
After applying all the above filters on a set of real LMA diagrams, the method of moving averages showed the greatest performance. The graphs smoothed by this method were spared from single emissions triggered by noise, while the real areas of amplitude bursts remained well recognizable, almost without losing their amplitude. At the same time, on the set of LF diagrams, all applied filters showed extremely low efficiency: the amplitude bursts significantly decreased or the bipolar portions of the peaks mutually extinguished. Therefore, in this work, it was decided not to apply to filter for LF diagrams.

For the convenience of compiling reports and monitoring the state of scanned objects in the long term, it was proposed to use a color zoning system for sections of section loss, dividing areas into green, yellow and red sections of the percentage of section loss. Levels of loss of the cross-section below the green value are not dangerous, below the yellow one is insignificant, and levels of loss of the cross-section above the red value are especially dangerous. To determine the level of the defect, it is necessary to identify the noise level on the diagram..

$$
\mathrm{NL}=\sum_{i=1}^{n}\left|y_{i}-f_{i}\right|
$$

$y_{i}$ - the real LMA value in $\mathrm{i}$-th point; $f_{i}$-the LMA value in $\mathrm{i}$-th point after filter; $\mathrm{n}$ - the number of points

The file analysis system consists of three modules: the two modules of detection of defect location (for LMA and LF diagram separately), the module of complex analysis of LMA and LF diagrams.

\subsection{The algorithm of module of detection of defect location on LMA diagram}

The algorithm consists of the following steps:

- One sets the minimum critical threshold for LMA (green level), bursts with its amplitude not exceeding it are not significant. Then one sets the yellow and red levels for detectable sections of cross-section loss.

- User sets the average noise level of the channel. In case this level is not set by the user, the level detected automatically by the equation (1).

- The algorithm sequentially scans all points of the signal along the increasing X coordinate.

- $\quad$ The first point found, whose amplitude exceeds the noise level, is recorded in a temporary array.

- If the recorded point is the beginning of the segment, the first point encountered is recorded in the temporary array, at which the amplitude reaches the noise level or falls below.

- If there is at least one point in the selected area that exceeds the established limits, then all points of this area are recorded in the structure, which is added to the array of detected sections of section loss.

\subsection{The algorithm of the module of detection of defect location on LF diagram}

The algorithm consists of the following steps:

- Creating an auxiliary array of points, the vertical values of which are taken modulo. This allows you to get rid of emissions in the negative coordinate and get a graph of entirely positive amplitudes.

- Identify the average noise level of the diagram.

- The algorithm sequentially scans all points of the signal along the increasing X coordinate.

- $\quad$ Recording in a temporary array of points, the amplitude of which exceeds the noise level.

- Recording in a temporary array point at which the amplitude reaches the noise level or fall below it.

- If there is at least one point in the selected area that falls within the established boundaries, record all points of this section into the structure and add it to the array of detected sections of the section loss.

\subsection{The module of complex analysis of LMA and LF diagrams}

The LF diagrams have a higher priority than the LMA diagrams. Therefore, the LF diagrams are analyzed first. Then, for each local defect LF diagram sought local defect LMA diagram. 
The input data of the module are the results of the work of the two previous modules. The output of the module is a set of technical characteristics of the scanning process (length of the recorded diagrams, calibration scan parameters, date and time of the scan, preset levels for the section loss channel, additional technical scan data, initial points of the channels), the number of LMA defects, and their division into criticality zones, the number of LF defects, the minimum, maximum and average values of the LMA diagrams, the minimum and maximum amplitude values for LF diagrams.

\subsection{The report output system}

The output of the module of complex analysis of LMA and LF diagrams is written into word or csv file. The graphic part of the report is formed using the pyplot library. The example of the graphic part of the report is shown in figure 3 .
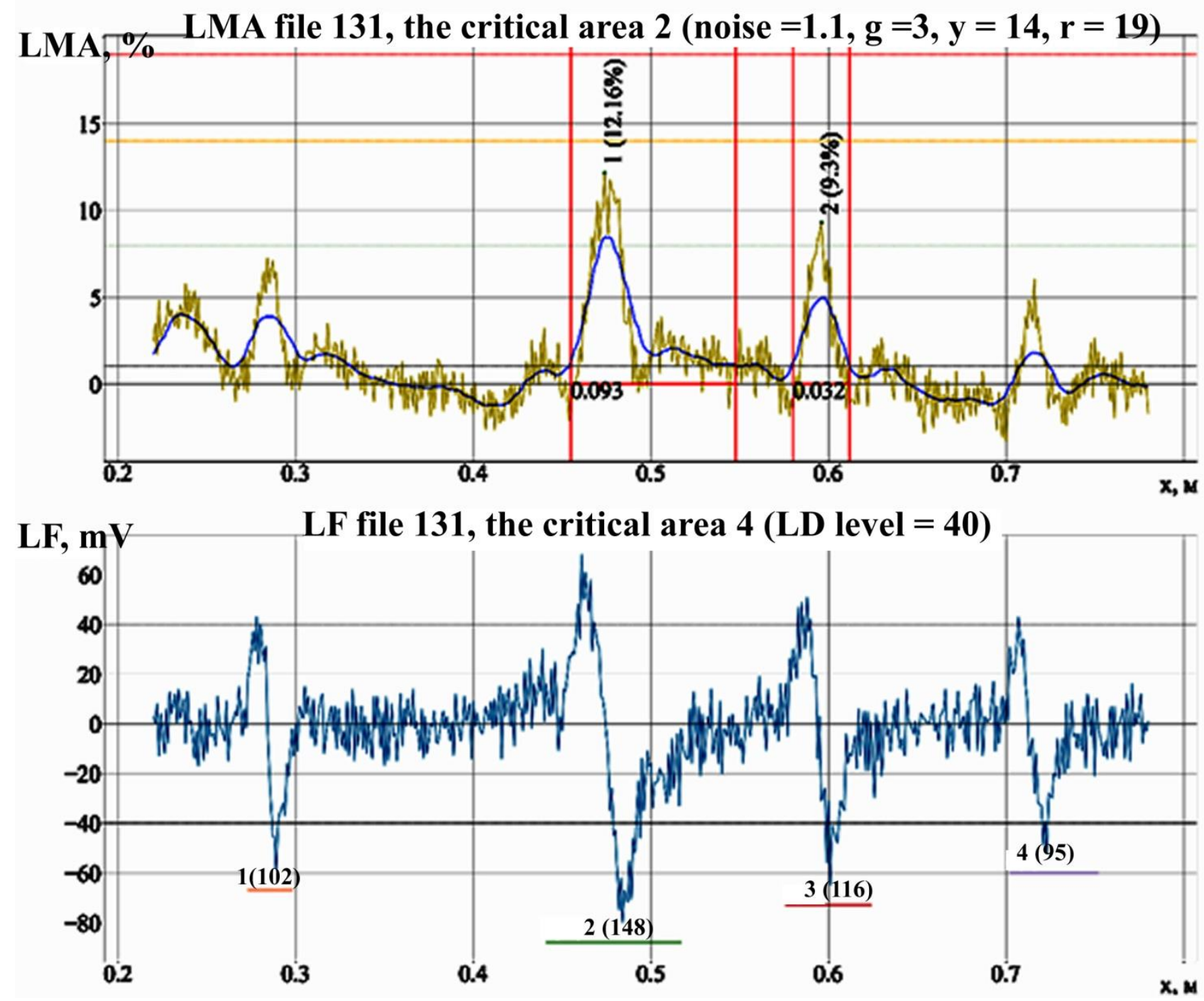

Fig. 3. The graphic part of report

\section{Conclusion}

We have developed the prototype of the system for complex analysis LMA and LF diagrams. We have tested it on laboratory test data. During testing, the system identified all defects applied to the ground wire and generated a report on them. Thus, we obtain the system for recognition of ground wire defects using the LMA and LF diagrams data. The system is ready for field testing.

This system will be used in the Cablewalker complex for diagnostics of faults of ground wires and wires of power lines along with computer vision systems. Using the system will detect not only visible but also hidden defects of the elements of power lines. 


\section{References}

[1] Breido, I.; Kaverin, V.; Ivanov, V.; Voytkevich, S. \& Levin, I. (2017). System of Protection and Diagnostics of Structural Elements of Support of High-Voltage Power Lines, Proceedings of the 28th DAAAM International Symposium, pp.0322-0330, B. Katalinic (Ed.), Published by DAAAM International, ISBN 978-3-902734-11-2, ISSN 1726-9679, Vienna, Austria DOI: 10.2507/28th.daaam.proceedings.044

[2] Huo, Yu., Prasad, G., Atanackovic, L., Lampe, L. \& Leung, V.C.M. (2019). Cable Diagnostics With PLMs for Smart Grid Monitoring, IEEE Access, Vol. 7, 2019, pp. 60206-60220, ISNN: 21693536, DOI: 10.1109/ACCESS.2019.2914580

[3] Qin, X., Wu, G., Lei, J., Fan, F., Ye, X. \& Mei, Q. (2018). A novel method of autonomous inspection for transmission line based on cable inspection robot lidar data, Sensors, Vol. 18, No. 2, 2018, pp. 1-22, ISSN: 14248220, DOI: 10.3390/s18020596

[4] Zhang, Y., Huang, X., Jia, J. \& Liu, X. (2019). A recognition technology of transmission lines conductor break and surface damage based on aerial image, IEEE Access, Vol. 7, 2019, pp. 59022-59036, ISSN: 21693536, DOI: 10.1109/ACCESS.2019.2914766

[5] Zhong, X. \& Zhang, X. (2016). Non-destructive testing of steel wire rope using magnetic flux leakage: Principle, sensor design and signal wavelet analysis, International Journal of Simulation: System, Science and Technology, Vol. 17, No. 26, 2016, ISSN: 14738031, DOI: 10.5013/IJSSST.a.17.26.26

[6] Sukhorukov, V.V., Slesarev, D.A. \& Vorontsov, A.N. (2014). Electromagnetic inspection and diagnostics of steel ropes: technology, effectiveness and problems, Materials Evaluation, Vol. 72, No. 8, 2014, pp.1019-1027, ISSN: 00255327

[7] http://cablewalker.com/en/, (2019). The Cablewalker 